Laser-induced magnetization dynamics in a cobalt/garnet heterostructure

This content has been downloaded from IOPscience. Please scroll down to see the full text. 2014 EPL 10527006

(http://iopscience.iop.org/0295-5075/105/2/27006)

View the table of contents for this issue, or go to the journal homepage for more

Download details:

IP Address: 131.174.17.24

This content was downloaded on 30/06/2014 at 12:32

Please note that terms and conditions apply. 


\title{
Laser-induced magnetization dynamics in a cobalt/garnet heterostructure
}

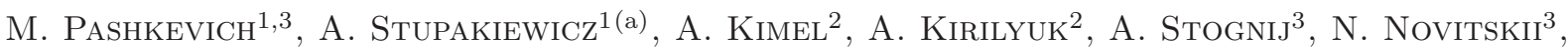 \\ A. Maziewsiri ${ }^{1}$ and Th. RAsing ${ }^{2}$ \\ 1 Laboratory of Magnetism, Faculty of Physics, University of Bialystok - Lipowa 41, 15-424 Bialystok, Poland \\ 2 Radboud University Nijmegen, Institute for Molecules and Materials - Heyendaalseweg 135, \\ 6525 AJ Nijmegen, The Netherlands \\ 3 Scientific-Practical Materials Research Centre of the NASB - P. Brovki 19, 220072 Minsk, Belarus
}

received 16 October 2013; accepted in final form 19 January 2014

published online 12 February 2014

PACS 75.78.Jp - Ultrafast magnetization dynamics and switching

PACS 78.20.Ls - Magneto-optical effects

PACS 75.50.Gg - Ferrimagnetics

\begin{abstract}
We report on magnetization dynamics triggered in a Co/garnet heterostructure by femtosecond laser pulses. Although laser excitation of a bare Co-doped yttrium iron garnet leads to magnetization precession, the phase of which strongly depends on the linear polarization of the light pulses, the deposition of an ultrathin Co layer on a top of a garnet film results in substantial changes of the laser-induced dynamics. The precession in the garnet is shown to lose its sensitivity to the polarization. Instead, light triggers polarization insensitive precession in both the magnetostatically coupled Co layer and the garnet film at two distinct frequencies typical for Co and garnet layers.
\end{abstract}

Copyright (C) EPLA, 2014

Introduction. - Manipulation of magnetization with the help of femtosecond laser pulses is a hot topic in fundamental science [1-4]. Understanding optical control of the magnetism in magnetic heterostructures is a particularly important issue for further development of faster magnetic information storage/processing and spintronic nanodevices. Optical control of spins in $\mathrm{Co} / \mathrm{SmFeO}_{3}$ heterostructures has been recently demonstrated using X-ray photoemission electron microscopy, revealing that the dynamics of the spins in the metallic $\mathrm{Co}$ and the dielectric $\mathrm{SmFeO}_{3}$ are correlated [5]. However, since the time resolution of these experiments was limited by the duration of the X-ray pulse (about $70 \mathrm{ps}$ ), these findings have raised the question whether the correlations between metallic and dielectric layers in such heterostructures are also present at the sub-100 ps time scale.

To investigate this issue we studied a similar system consisting of a dielectric yttrium iron garnet (YIG) film and an ultrathin Co layer. It is known that in such a heterostructure one can observe a strong influence of a $2 \mathrm{~nm}$ Co layer on both the domain structure and magnetization

(a) E-mail: and@uwb.edu.pl reversal processes in the garnet, due to magnetostatic coupling [6]. A priori, one can anticipate several mechanisms which can be responsible for possible correlations between the magnetization dynamics in the Co and garnet layers of such a heterostructure. For instance, it is known for a metal/dielectric heterostructure that spin-orbital interaction may initiate a transfer of angular momentum between the layers, and thus cause correlations in the magnetization dynamics [7]. In addition, it can occur as a consequence of exchange coupling, such as has been found in a Py/YIG bilayer [8]. The goal of the research reported in this paper is to understand whether the ultrathin Co film also affects laser-induced magnetization dynamics in the garnet film.

Here we compare laser-induced magnetization dynamics in a cobalt/garnet heterostructure with that observed for the bare garnet thin film. Although optical excitation of a bare garnet leads to magnetization precession, the phase of which strongly depends on the polarization of the pump light, the deposition of an ultrathin Co layer on top of the garnet film is shown to lead to substantial changes of the laser-induced dynamics. The precession becomes insensitive to the polarization of the laser pulse and occurs 


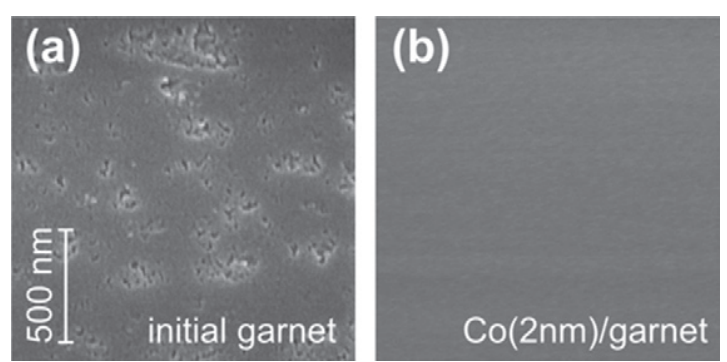

Fig. 1: Scanning electron microscopy images of the initial garnet surface before etching (a) and after etching and subsequent deposition of $2 \mathrm{~nm}$ Co (b).

in both the Co layer and the garnet film, at two distinct frequencies typical for an ultrathin Co layer and a garnet film, respectively. The results reveal the existence of ultrafast correlations between the spins of the magnetic layers.

Experimental details. - We studied a heterostructure obtained by the growth of a $2 \mathrm{~nm}$ Co polycrystalline layer on a $1.8 \mu \mathrm{m}$ Co-doped YIG film (YIG:Co). Scanning electron microscopy images of the surface of the sample are shown in fig. 1. The original $6.5 \mu \mathrm{m}$ garnet had a rough surface with protrusions about $100-200 \mathrm{~nm}$ in diameter (see fig. 1(a)), which was thinned down with a $0.6 \mathrm{keV}$ oxygen ion beam and smoothed using a $0.3 \mathrm{keV}$ beam for over 10 minutes. After the reduction of the thickness from $6.5 \mu \mathrm{m}$ to $1.8 \mu \mathrm{m}$ and the subsequent etching, we also obtained a significant reduction of the surface roughness with an improvement of the root-mean-square roughness from 3.5 to $0.3 \mathrm{~nm}$ (see fig. 1(b)). The formation of continuous Co layers on a smoothed garnet surface is caused by irradiation of high-energy atoms from a sputtered flux [9]. High-energy atoms penetrate deep in the garnet film and in the growing Co layer. At the initial stage of Co growth, the atoms of the sputtered flux improve the adhesion of the Co layer to the smoothed garnet surface and favor continuity in the thinner layers as a result of compacting the growing layer during atomic collisions. The Co/garnet interface is sharp, and the thickness of the transition layer is thinner than $1 \mathrm{~nm}$. Finally, a $4 \mathrm{~nm} \mathrm{Au}$ film was used to protect the Co layer from oxidation. At room temperature the magnetic anisotropy constants of the $1.8 \mu \mathrm{m}$ thick garnet film were $K_{1}=-2 \times 10^{3} \mathrm{erg} / \mathrm{cm}^{3}$ and $K_{\mathrm{u}}=10^{3} \mathrm{erg} / \mathrm{cm}^{3}[6]$, and Gilbert damping was 0.19. For the $2 \mathrm{~nm}$ Co layer, the effective anisotropy constant and Gilbert damping were $K_{\mathrm{eff}}^{\mathrm{Co}}=-9.9 \times 10^{6} \mathrm{erg} / \mathrm{cm}^{3}$ and 0.04 , respectively $[6,10]$.

To study the ultrafast spin dynamics in the bilayer structure induced by femtosecond laser pulses, we carried out time-resolved measurements at room temperature using a magneto-optical pump-probe method. Pump pulses with a duration of $35 \mathrm{fs}$ from a Ti:Sapphire laser system with an amplifier (Ace, Spectra-Physics) at a $500 \mathrm{~Hz}$ repetition rate and wavelength of $800 \mathrm{~nm}$ were directed at
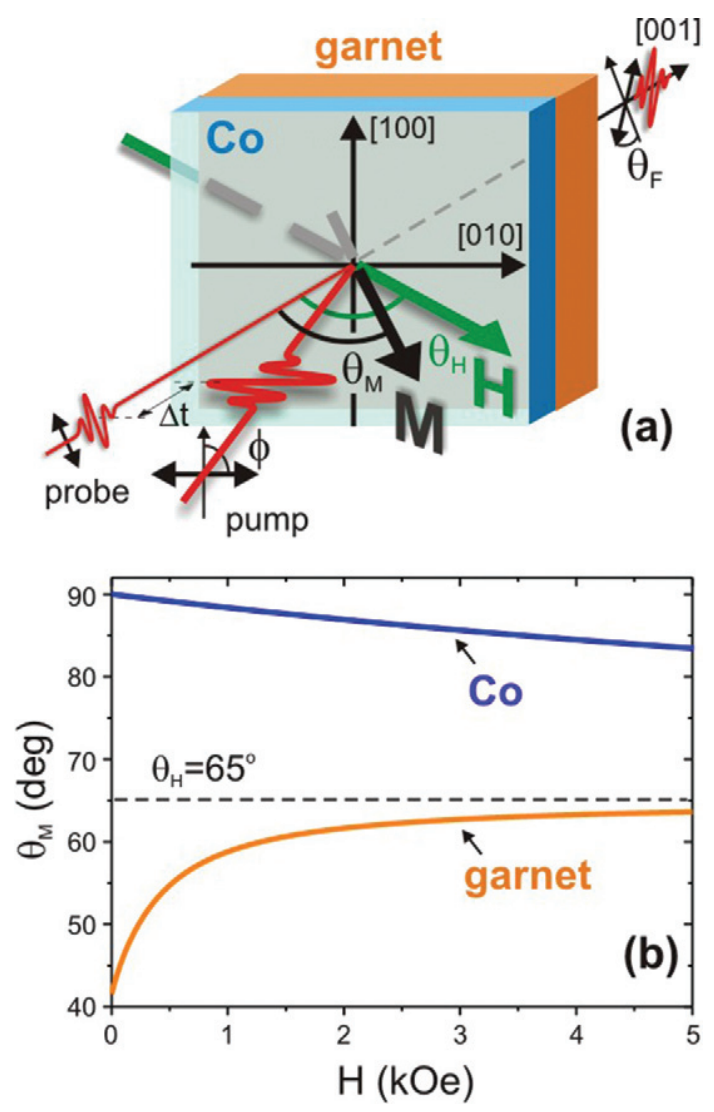

Fig. 2: (Colour on-line) Experimental geometry (a) and magnetization orientation as a function of the amplitude of external field $H$ for garnet and Co layer of the heterostructure (b).

an angle of incidence about $10^{\circ}$ from the sample normal parallel to the [001] crystallographic axis of the garnet film. The pump beam with a fluence of $10 \mathrm{~mJ} / \mathrm{cm}^{2}$ was focused onto a spot of about $100 \mu \mathrm{m}$ in diameter. The sample was excited by the pump through the Co side of the heterostructure. The probe beam was about two times smaller in size and intensity than the pump. The probe pulses at a $1 \mathrm{kHz}$ repetition rate and wavelength of $800 \mathrm{~nm}$ were directed perpendicular to the sample plane, see fig. 2(a). The delay time $\Delta t$ between the pump and the probe pulses could be adjusted up to $2 \mathrm{~ns}$. The polarization plane of the linearly polarized pump pulse was adjusted to an angle $\phi$ with respect to the [100] axis. The polarization plane of the probe beam was along the [1]10] axis. We probed the dynamics of the perpendicular component of the magnetization $M_{z}$ measuring the Faraday rotation angle $\theta_{\mathrm{F}}$ of the probe as a function of the delay time between the pump and probe pulses $\Delta t$.

Results and discussion. - A rather unique combination of magnetic properties of the layers allows us to realize different regimes of the laser-induced dynamics. Changing the strength of the magnetic field we were able to obtain conditions when the magnetization dynamics was dominated either by the Co or the garnet layer. Such a layer 
selective probing of the magnetization dynamics can be understood by a simple phenomenological model [6]. Assume that our bilayer system is in an external magnetic field $H$ applied at an angle $\theta_{\mathrm{H}}=65^{\circ}$ so that the layers are in a monodomain state and the layers are characterized by two magnetizations vectors $M_{\text {garnet }}$ and $M_{\mathrm{Co}}$.

The equilibrium orientation of the magnetizations in the Co and garnet layers can be found by minimizing the free energy of the total system. The expression for the free energy contains terms representing the energy of the magnetic anisotropies, the Zeeman energy in the external magnetic field $H$ as well as the demagnetization energies defined by the saturation magnetizations for the Co and the garnet layers [6]. Figure 2(b) shows how the angle $\theta_{\mathrm{M}}$, between the normal to the sample and the equilibrium orientations of the magnetizations in the layers, depends on the external magnetic field $H$. In our Faraday geometry, the probe pulse polarization rotation is proportional to the magnetization component along direction normal to the sample plane. The angle $\theta_{\mathrm{F}}$ of the probe oscillates as a fingerprint of the spin precession. Therefore, increasing the angle $\theta_{\mathrm{M}}$ leads to increasing the amplitude of the spin oscillations. It is seen from the figure that if an ultrashort laser excitation triggers magnetization dynamics of small amplitude, we can clearly distinguish two regimes: i) the low-magnetic-field regime $(H<1 \mathrm{kOe})$ when the laser-induced dynamics is dominated by the magnetization precession of the garnet film due to the simultaneity of the large angle between $H$ and $M_{\text {garnet }}$, and close-tozero perpendicular component of $M_{\mathrm{Co}}$; ii) the high-field regime $(H>4 \mathrm{kOe})$, when the laser-induced dynamics is dominated by the magnetization precession of the $\mathrm{Co}$ film with the significant perpendicular component $M_{\mathrm{Co}}$, on conditions that the magnetization vector $M_{\text {garnet }}$ practically is along $H$.

Time dependences of the $z$-component of the $M_{z}$ magnetization component were thus measured for different orientations of the linear polarization of the pump pulse and at different external magnetic fields $H$. Figure $3($ a) shows the magnetization dynamics measured at $1.5 \mathrm{kOe}, 2.3 \mathrm{kOe}$ and $4.6 \mathrm{kOe}$ for the polarization of the pump $\phi=0^{\circ}$. It is seen that the laser excitation triggers oscillations of the signal at two frequencies which are idenified as the frequencies of the ferromagnetic resonance in the Co and the garnet layers, respectively [10]. It is also seen that upon increasing the magnetic field, the contribution from the garnet vanishes so that at a field of $4.6 \mathrm{kOe}$ the contribution from the Co layer dominates. From the experimental curves, we deduced amplitudes of the oscillations using fit with a sinusoidal function with damping (fig. 3(b)). At the field of $4.6 \mathrm{kOe}$ we have also measured the laser-induced dynamics as a function of the polarization of the pump, setting the latter at $\phi=0^{\circ}$ and $90^{\circ}$ (see fig. 3(c)). In this case we observe no polarization dependence of the oscillations. In general, the laser-induced precession can be excited due to an ultrafast laser-induced demagnetization typical for metallic magnets [11]. The demagnetization is
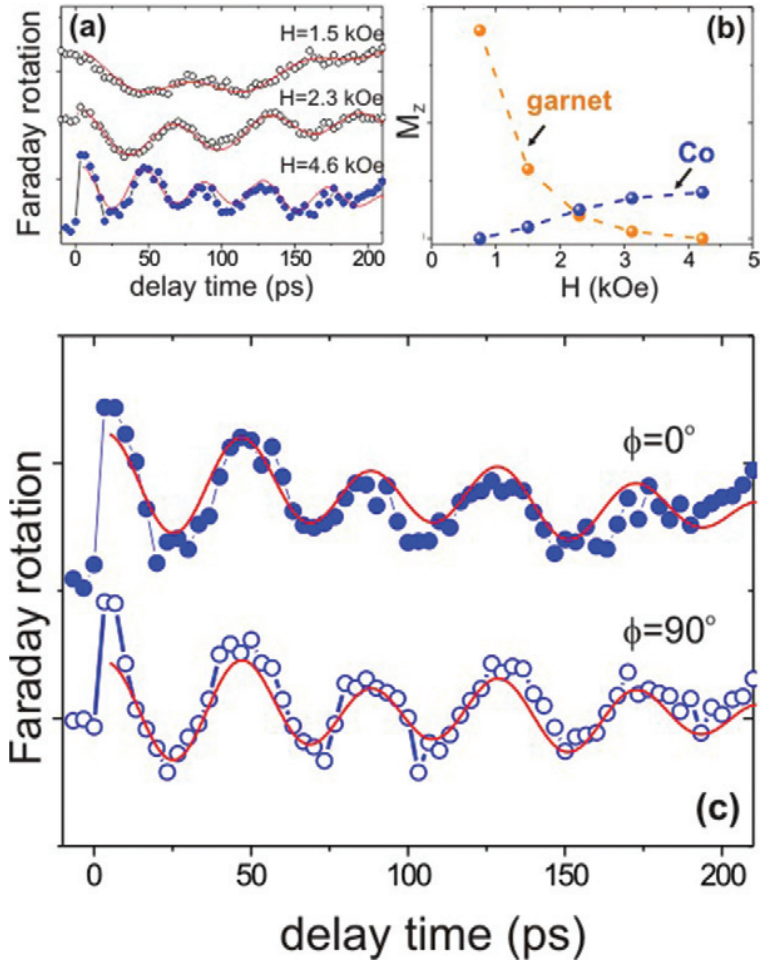

Fig. 3: (Colour on-line) Time-resolved Faraday rotation of the $\mathrm{Co} /$ garnet film as a function of the delay time $\Delta t$ for different magnetic-field amplitude (a) and pump polarization (b). Dependence of the magnetization component $M_{z}$ on the amplitude of external field $H$ for the garnet and Co layer (b). Solid lines were fitted using the classical oscillation function including damping for a $2 \mathrm{~nm}$ Co layer on a $1.8 \mu \mathrm{m}$ garnet film [10].

seen as a sub-picosecond change of the magneto-optical signal measured at $H=4.6 \mathrm{kOe}$. Such an ultrafast demagnetization leads to an ultrafast reduction of $M_{\mathrm{Co}}$, which effectively changing the equilibrium orientation of the magnetization in this layer and thus triggering spin oscillations $[12,13]$. In contrast to the laser-induced magnetization dynamics triggered in the bare garnet film, it is clearly seen that the laser-induced magnetization dynamics in the Co layer is polarization independent. Hence, the magnetization excited in the Co layer is typical for metallic magnets and thus does not seem to be affected by the vicinity of the garnet film.

In order to investigate whether the Co layer affects the magnetization dynamics in the garnet film, we performed time-resolved measurements of the laser-induced magnetization dynamics in the heterostructure in the low-field regime $H<1 \mathrm{kOe}$. In this regime the garnet magnetization precession amplitude always dominates that of the cobalt layer [10]. First we measured the laser-induced magnetization dynamics in a bare garnet film. Figure 4 shows that changing the polarization of the pump induces a shift of the phase of the precession $\Delta \psi$ in the bare garnet film (see fig. 4(a)). 


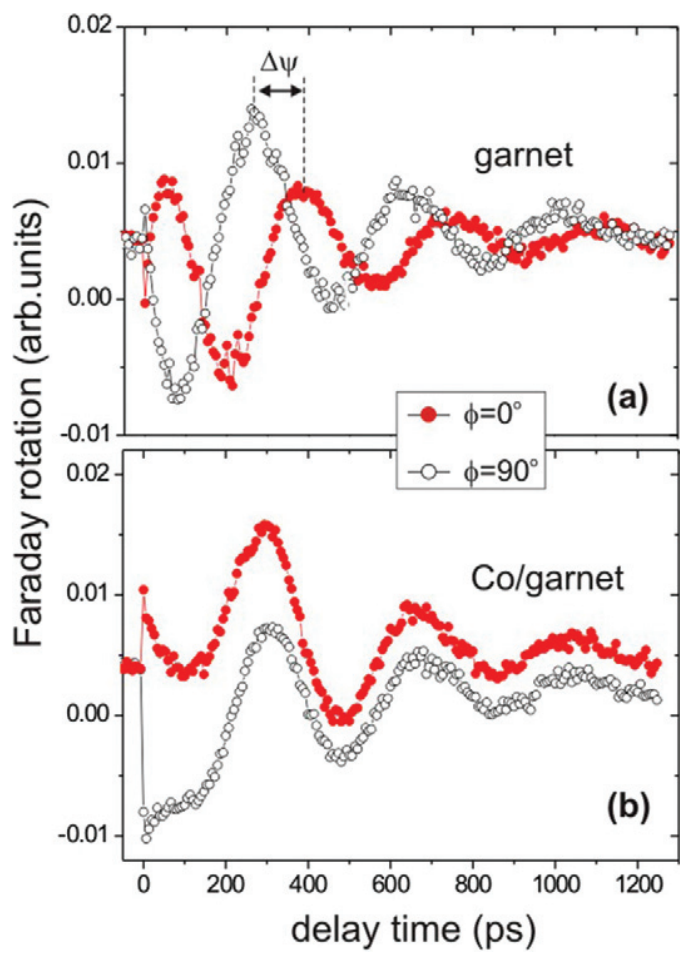

Fig. 4: (Colour on-line) Time-resolved Faraday rotation of garnet and $\mathrm{Co} /$ garnet films as a function of the delay time $\Delta t$ for a magnetic field $H\left(\theta_{\mathrm{H}}=65^{\circ}\right)=0.75 \mathrm{kOe}$ and different pump polarization $\phi$.

This is in agreement with earlier reports on laserinduced magnetization dynamics in garnet films [14]. However, after the deposition of the nanometer-thin Co film, the sensitivity of the laser-induced magnetization dynamics to the polarization vanishes. Pump pulses polarized at $\phi=0^{\circ}$ and $90^{\circ}$ trigger magnetization dynamics in the garnet film with the very same phase $\Delta \psi=0$, see fig. 4(b). At the time scale up to $100 \mathrm{ps}$ the magnetization dynamics triggered in the bare garnet and $\mathrm{Co} /$ garnet films with the same polarization of the pump light are clearly different. At the same time, the frequencies of the precession in these two samples are practically the same.

The effect of the Co layer on the laser-induced dynamics in the garnet film can be explained as follows. The laser excitation leads to both an ultrafast demagnetization of the Co layer and a photoinduced magnetic anisotropy in the garnet film. These effects lead to a rapid change of the equilibrium orientations of the magnetizations in the layers given by the external out-of-plane magnetic field, the demagnetizing factor, effective field of the photo-induced anisotropy in the garnet and the effective magnetocrystalline anisotropy field in the Co/garnet bilayer. Such a rapid change triggers precession of the spins around the new equilibrium orientation. In a Co-doped garnet film, the initial phase of the spin precession depend on whether the pump laser pulse is polarized parallel to the [100] or [010] crystallographic axes, which define the local tetragonal symmetry. In this case, the polarization-dependent ex- citation of the precession leads to a change of the magnetic anisotropy in the garnet due to the uniaxial photo-induced anisotropy [14]. The direction of this photo-induced anisotropy field is defined by the pump pulse polarization. At the initial time after the pump pulse, the magnetization decays with a characteristic time of about 20 ps [14]. However, in a $\mathrm{Co} /$ garnet heterostructure at low applied field $(H<1 \mathrm{kOe})$, the phase of the spin precession is not only defined by the internal, photo-induced anisotropy fields, but also by the magnetostatic stray field produced by the Co layer $\left(M_{\mathrm{Co}} \gg M_{\text {garnet }}[6]\right)$. The magnetostatic interlayer coupling is responsible for a change in phase of the magnetization precession in the neighboring soft garnet film. Thus, in a heterostructure, during $60 \mathrm{ps}$ the magnetization partially recovers and the recovery is accompanied by a precession of the magnetization around the effective magnetic field. The direction component of this field is isotropic in the sample plane due to the easy plane character of the magnetic anisotropy of the $2 \mathrm{~nm}$ Co film. Furthermore, the amplitude of the effective photo-induced anisotropy field is rather small [14] compared to the effect originating from a change of the demagnetizing field due to the ultrafast laser-induced demagnetization of Co. Hence the excitation of the magnetization precession is a result of the demagnetization of Co.

In conclusion, we investigated the ultrafast magnetization dynamics of a Co/YIG:Co heterostructure. To explore the effect of light on the observed precessions, we studied the dependence of the spin dynamics in the heterostructure on the pump polarization and the external magnetic field. It is shown that the excitation of the garnet with linearly polarized laser pulses triggers a polarizationsensitive magnetization precession in the bare garnet film. The mechanism that triggers the precession is a photoinduced change of the magnetic anisotropy. However, a growth of just a $2 \mathrm{~nm}$ Co film on top of the garnet significantly changes the mechanism of the laser-induced precession in the heterostructure. In particular, the light triggers polarization-independent precession in both the Co and garnet layers via the magnetostatic coupling between these layers.

$$
* * *
$$

This work was supported by National Science Centre Poland for OPUS project DEC-2013/09/B/ST3/02669.

\section{REFERENCES}

[1] Beaurepaire E., Merle J.-C., Daunois A. and Bigot J.-Y., Phys. Rev. Lett., 76 (1996) 4250.

[2] Koopmans B., Malinowski G., Dalla Longa F., Steiauf D., Fähnle M., Roth T., Cinchetti M. and Aeschlimann M., Nat. Mater., 9 (2010) 259.

[3] Bovensiepen U., Nat. Phys., 5 (2009) 461.

[4] Kirilyuk A., Kimel A. V. and Rasing Th., Rev. Mod. Phys., 82 (2010) 2731. 
[5] Le Guyader L., Kleibert A., Nolting F., Joly L., Derlet P. M., Pisarev R. V., Kirilyuk A., RasING Th. and Kimel A. V., Phys. Rev. B, 87 (2013) 054437.

[6] Pashkevich M., Stupakiewicz A., Kirilyuk A., Maziewski A., Stognis A., Novitskit N., Kimel A. and Rasing Th., J. Appl. Phys., 111 (2012) 023913.

[7] Woltersdorf G., Mosendz O., Heinrich B. and Back C. H., Phys. Rev. Lett., 99 (2007) 246603.

[8] Vukadinovic N., Ben Youssef J., Castel V. and Labrune M., Phys. Rev. B, 79 (2009) 184405.

[9] Stognil A. I., Pashkevich M. V., Novitskit N. N., Gribkov B. A., Mironov V. L., Ketsko V. A., FetTAR F. and GARAD H., Inorg. Mater., 45 (2009) 1240.
[10] Stupakiewicz A., Pashkevich M., Maziewski A., Stognid A. and Novitski N., Appl. Phys. Lett., 101 (2012) 262406.

[11] Kisielewski J., Kirilyuk A., Stupakiewicz A., Maziewski A., Kimel A., Rasing Th., Baczewski L. T. and Wawro A., Phys. Rev. B, 85 (2012) 184429.

[12] Bigot J.-Y., Vomir M., Andrade L. H. F. and BeauRepaire E., Chem. Phys., 318 (2005) 137.

[13] Vomir M., Andrade L. H. F., Guidoni L., BeaurePaire E. and Bigot J.-Y., Phys. Rev. Lett., 94 (2005) 237601.

[14] Atoneche F., Kalashnikova A. M., Kimel A. V., Stupakiewicz A., Maziewski A., Kirilyuk A. and RAsing T., Phys. Rev. B, 81 (2010) 214440. 